\title{
Ecología y fitosanidad de los encinos (Quercus spp.) en la Sierra Fría, Aguascalientes, México
}

\author{
Ecology and phytosanitary condition of oak trees in the \\ (Quercus spp.) in the Sierra Fría range, Aguascalientes, Mexico
}

\author{
Joaquín Sosa-Ramírez1, Onésimo Moreno-Rico2, \\ Guillermo Sánchez-Martínez³, María Elena Siqueiros-Delgado2 \\ y Vicente Díaz-Núñez 4
}

\begin{abstract}
RESUMEN
Durante los años 2007, 2008 y 2009 se condujo un estudio para evaluar la distribución y abundancia de las especies de encinos (Quercus spp), los factores ambientales que afectan su distribución y la identificación de los patógenos e insectos asociados a la declinación y muerte de estos árboles en la Sierra Fría, Aguascalientes. Para evaluar la distribución y abundancia se realizaron 60 inventarios de la vegetación en 60 sitios de muestreo distribuidos al azar utilizando parcelas de $600 \mathrm{~m}^{2}$. Para la identificación de los fitopatógenos se hicieron 28 transectos, revisando 100 árboles en cada uno. Para la identificación de los insectos asociados con la declinación de los encinares se instalaron trampas de emergencia sobre el fuste de árboles con síntomas de ataque de insectos barrenadores y se tomaron muestras de fuste, las cuales se observaron en laboratorio. Se identificaron 10 especies de encinos, siendo la altitud, el relieve, la exposición del sitio y la fisiografía las variables que influyeron sobre su distribución y abundancia. Se identificaron los fitopatógenos Phellinus robustus, $P$. gilvus, $P$. everhartii, Ganoderma lucidum e Hypoxylon thouarsianum, siendo el último el más ampliamente distribuido. Se identificó al barrenador Crioprosopus magnificus infestando encinos vivos. La información obtenida contribuye al manejo de la Sierra Fría y se sugiere profundizar en estos estudios.
\end{abstract}

PALABRAS CLAVE:

Cerambycidae, encinos, enfermedades forestales, Sierra Fría.

\begin{abstract}
A study was conducted during 2007, 2008 and 2009 to evaluate the distribution and abundance of oak species (Quercus spp), the environmental factors which affect their distribution, and the identification of pathogens and insects associated with oak decline and mortality in the Sierra Fria, Aguascalientes. To evaluate oak distribution and abundance, 60 plant inventories were made in 60 sampling sites randomly distributed within the landscape, using $600 \mathrm{~m}^{2}$ plots. To identify plant pathogens 28 transects were established, inspecting 100 oak trees per transect. To identify insects associated with oak decline, emergence traps were installed on the bole of trees that show wood boring symptoms; bole sections were taken to the lab for beetle capture. Ten oak species were identified and the variables that have influence on their distribution and abundance, were elevation, relief, aspect and phys-
\end{abstract} Centro de Ciencias Básicas. Universidad Autónoma de Aguascalientes. Omoreno@correo.uaa.mx, masiquei@correo.uaa.mx

3 INIFAP-CIRNOC-Campo Experimental Pabellón. sanchezm.guillermo@inifap.com.mx

4 Biodiversidad, Recursos Forestales y Suelos. Secretaría de Medio Ambiente del Estado de Aguascalientes. vdiaz_ing@yahoo.com.mx. 
iography. As plant pathogens we identified Phellinus robustus, $P$. gilvus, $P$. everhartii, Ganoderma lucidum and Hypoxylon thouarsianum, the last one being the more widely distributed. The oak borer Crioprosopus magnificus was identified, infesting live oak trees. The presence of this insect is a new report for the area of study. The information gathered in this study contributes to the management of the Sierra Fría and further studies are suggested.

KEY WORDS:

Cerambycidae, oak trees, forest diseases, Sierra Fria.

\section{INTRODUCCIÓN}

En los ecosistemas templados de montaña las especies de Pinus y Quercus son ampliamente dominantes en las comunidades de plantas leñosas. El género Quercus presenta la más amplia distribución a nivel mundial. Se reconocen dos centros de diversidad, el primero localizado en el sureste de Asia, alberga poco más de 120 especies (Govaerts y Frodin, 1998) y el segundo centro de diversidad genética es México, principalmente en las regiones montañosas, donde comparte espacios con comunidades de coníferas (Valencia, 2004). En México el número señalado de especies de encinos es variable, Nixon (1993) refiere la existencia de entre 135 y 150; por su parte, Valencia (2004) sugiere que son 161. Los encinos son de excepcional valor por la calidad de su madera, así como por múltiples usos y productos, entre otros: leña, carbón, corcho, taninos y colorantes, alimento para el hombre y el ganado (Espejel-Rodríguez et al., 1999).

A pesar de su importancia, los ecosistemas templados donde se ubican los encinares están disminuyendo, ya sea por causas naturales o debido a la inter- vención del hombre. Por ejemplo, áreas que originalmente estaban cubiertas por encinos han sido desplazadas por las actividades agropecuarias (Challenger, 1998). Por otro lado, en México, desde hace varios años se ha observado una muerte de encinos en diferentes estados, principalmente en Colima, Guanajuato, Jalisco, Nayarit y Aguascalientes (Tainter et al., 2000; Kliejunas, 2005). La literatura sobre este problema en México es escasa. Tainter et al. (2000) señalan al oomiceto Phytophthora cinnamomi Rands., como el principal agente causal de la muerte de encinos en el estado de Colima. En el mismo estado, Alvarado et al. (2007) aislaron a P. cinnamomi de Quercus elliptica, Q. salicifolia y $Q$. acutifolia. Además, Alvarado et al. (2007) confirmaron que $P$. cinnamomi fue el causante de muertes masivas de encinos en Teconapa, Guerrero, siendo los más dañados $Q$. elliptica y $Q$. salicifolia. Asimismo, en la Sierra de Lobos, Guanajuato, Vázquez et al. (2004) encontraron que la combinación de los hongos ascomicetos Nectaria galligena e Hypoxylon thouarsianum fueron los que causaron más muerte en encinos. En el estado de Aguascalientes, desde hace varios años se ha estado observando enfermedad y muerte de encinos. Antes de morir los árboles presentan muerte descendente de las ramitas, amarillamiento, muerte y caída de las hojas y presencia de cancros en el tallo y ramas. Al respecto, Alvarado et al. (2007), realizaron estudios sobre el declinamiento de encinos en Aguascalientes, Colima, Guanajuato, Jalisco y Nayarit. En el caso de la Sierra Fría, en Aguascalientes, reportaron la presencia del hongo ascomiceto Hypoxylon atropunctatum y de la planta superior parásita Phoradendron villosum afectando a encinos y al oomiceto Pythium en el suelo. A pesar de lo anterior, es importante conocer si existen otros agentes causantes de enfermedad y muerte de encinos en dicha zona. 
Referente a los insectos coleópteros barrenadores de encinos vivos en México, Cibrián et al. (1995) mencionan a Tylcus hartwegii (White) (Coleoptera: Cerambycidae) que se alimenta de ramas de Quercus spp., en el estado de Nuevo León, algunas especies no identificadas de Agrilus (Coleoptera: Buprestidae) que se alimentan de ramas de hasta $8 \mathrm{~cm}$ de diámetro. En el este de Estados Unidos, el barrenador del encino rojo (Enaphalodes rufulus Haldeman) (Coleoptera: Cerambycidae) ataca encinos vivos y participa en el complejo de factores contribuyentes a la declinación y muerte de los encinos (Lawrence et al., 2002; Heitzman et al., 2007). Durante el presente estudio, en 2006 se detectaron síntomas de barrenadores afectando encinos vivos en la Sierra Fría, Aguascalientes, razón por la cual se abordó su estudio.

\section{OBJETIVOS}

Caracterizar la distribución y abundancia de las comunidades de encinos en un área de la Sierra Fría, Aguascalientes.

Identificar a los agentes patógenos causantes de la muerte de los encinos y la distribución espacial de la enfermedad.

Conocer el agente causal de barrenación de los encinos en la Sierra Fría, Aguascalientes.

\section{MATERIALES Y METODOLOGÍA}

El estudio abordó tres aspectos: la distribución y abundancia de las comunidades de encino, los microorganismos patógenos asociados y la detección de plagas que afectan a los encinares. El estudio se realizó dentro de la zona forestal del Área Natural Protegida Sierra Fría y algunos sitios circunvecinos.

\section{Distribución y abundancia de especies de encinos}

Para caracterizar la distribución y abundancia de las especies de encinos se seleccionó un polígono de 25 mil hectáreas, representativo de la vegetación arbóreo-arbustiva de la Sierra Fría, ubicado en las coordenadas 102 $31^{\prime} 31^{\prime \prime}$ a $102^{\circ} 37^{\prime} 44^{\prime \prime}$ Longitud Oeste y $22^{\circ} 05^{\prime} 47^{\prime \prime}$ a $22^{\circ} 14^{\prime} 03^{\prime \prime}$ Latitud Norte. En el marco de este polígono se elaboró un plan de muestreo estratificado. Los estratos considerados fueron: altitud, geoforma y exposición. El estrato de la altitud se determinó usando cuatro clases: (2200$2400,2400-2600,2600-2800$ y > 2800) msnm; las geoformas consistieron en sitios cóncavos y convexos; para la exposición del terreno se contemplaron las orientaciones $\mathrm{N}, \mathrm{S}, \mathrm{E}, \mathrm{O}$ y sus combinaciones. Se realizaron 60 inventarios de la vegetación en igual número de sitios ecológicos, mismos que fueron determinados al azar. Los muestreos se realizaron en parcelas rectangulares de 600 $\mathrm{m}^{2}$, con una línea central de $100 \mathrm{~m}$ de longitud y dos líneas laterales de $3 \mathrm{~m}$ cada una. En cada uno de los inventarios se determinó la frecuencia de las especies existentes, midiendo tanto variables dasométricas convencionales de la vegetación, como variables ambientales. Los árboles fueron aquellos individuos con un diámetro a la altura del pecho (DAP) mayor o igual a $5 \mathrm{~cm}$ y altura mayor o igual a $1,50 \mathrm{~m}$. Las variables ambientales consideradas incluyeron la altitud sobre el nivel del mar (asnm), la pendiente (en \%), la exposición del terreno (N, S, E, W), la fisiografía (planicie, lomerío, meseta, ladera media, ladera alta, fondo de barranco, arroyo) y la geoforma del sitio. A la par se registraron variables de manejo, tales como el tipo de utilización del terreno y la intensidad del aprovechamiento, variables consideradas como responsables de la distribución de varias especies forestales. Cada uno de los 
puntos muestreados fue georreferido en Unidades Transversales de Mercator (UTM) y plasmado en una imagen del satélite $\operatorname{Spot} \circledast 2$ 2003. La abundancia de cada una de las especies de encino encontradas se determinó registrando sobre una línea de 100 puntos el número de veces que una especie aparece, respecto a todas las especies de árboles encontradas en el transecto. La frecuencia relativa fue calculada de acuerdo con Krebs (1993) como se especifica a continuación:

$$
f_{r e l}=\frac{f\left(x_{1}\right)}{\sum_{i=1}^{n} f(x 1)} \cdot 100
$$

Donde:

$\mathrm{f}_{\text {rel }}=$ Frecuencia relativa

$f\left(x_{i}\right)=$ Frecuencia de la especie

$x_{i}=$ frecuencia absoluta obtenida en los

muestreos

$\mathrm{n}=$ Número total de especies

Posteriormente se generó un índice de abundancia usando la fórmula:

Donde:

$$
I A_{s p p}=\frac{\sum \text { frel }}{m}
$$

$\mathrm{IA}_{\text {Spp. }}=$ Índice de abundancia de cada uno de los encinos identificados

$\mathrm{M}=$ Número de sitios muestreados

\section{Distribución de las enfermedades, identificación e incidencia de los fitopatógenos}

Durante julio de 2007 a marzo de 2009 se realizaron 28 recorridos de estudio en diferentes localidades de la Sierra Fría, Aguascalientes. En cada uno de los sitios visitados se realizó un transecto, de dimensiones variables, en una dirección determinada al azar, que incluyó la revisión de 100 árboles y la identificación de fitopatógenos y enfermedades que causan. Los porcentajes de incidencia de árboles dañados por los diferentes organismos fitopatógenos $u$ oportunistas se obtuvieron contando el número de árboles afectados a lo largo del transecto de dimensiones variables, que tuvo como límite 100 árboles.

La identificación de los fitopatógenos se basó en los síntomas y signos, basidiomas y ascomas, presentes en los árboles enfermos. Para los hongos poliporoides, en el laboratorio se emplearon las técnicas tradicionales propuestas por Gilbertson y Ryvarden (1986), Gilbertson y Ryvarden (1987) y Ryvarden (1991). Para la identificación de los hongos ascomicetos se tomaron las características de los estromas y ascomas señaladas por Ju-Ming y Rogers (1996) y Swiecki y Bernhardt (2006). La descripción de síntomas y signos encontrados en los encinos fueron comparados con los reportados en la bibliografía especializada para la identificación del agente causante de las enfermedades (Gilbertson y Ryvarden, 1986; Gilbertson y Ryvarden 1987; Ryvarden, 1991, 2000; Ju-Ming y Rogers, 1996; Swiecki y Bernhardt, 2006; Cibrián et al. 2007).

\section{Determinación del agente causal de barrenación en encinos}

En octubre de 2006 se detectaron los primeros síntomas de un barrenador de encinos en el área de estudio. El 13 de noviembre de 2006 se derribaron dos árboles con síntomas de barrenación activa en "La Angostura" (22 $05^{\prime} 57^{\prime}$ " N y $\left.102^{\circ} 41^{\prime} 49^{\prime \prime}\right)$. El fuste se seccionó con una motosierra, encontrando dos larvas de Cerambycidae. Con base en lo anterior, 
durante 2007 a 2009 se instalaron trampas de emergencia, construidas con malla mosquitera pegadas al fuste de árboles con síntomas de ataque de un barrenador, para capturar especímenes adultos y proceder a su identificación, Además de instalar trampas en el sitio de detección inicial de los síntomas, se mantuvieron trampas dentro del Centro de Educación Ambiental e Investigación "Los Alamitos", ubicado a 22 $10^{\prime} 28^{\prime \prime} \mathrm{N}$ y $102^{\circ} 35^{\prime} 15^{\prime \prime}$ W y en el predio "La Sauda", ubicado a $21^{\circ} 58^{\prime} 53^{\prime \prime} \mathrm{N}$ y $102^{\circ} 34^{\prime} 6^{\prime \prime}$ W (Sánchez-Martínez et al., 2010). La frecuencia de revisión de las trampas en los tres años fue quincenalmente durante el mes de mayo, semanalmente durante junio y quincenalmente durante julio $y$ agosto, tomando como referencia la biología de E. rufulus (Fierke et al., 2005) y Crioprosopus magnificus (Solomon, 1995). En complemento, cada año se tomaron muestras de secciones de fuste de encinos infestados, las cuales se mantuvieron en observación bajo condiciones de laboratorio, buscando obtener especímenes adultos. Para la identificación de la especie de insecto barrenador se utilizó literatura especializada, entre esta citamos a Hovore (1983) y Solomon (1995) y se contó con el apoyo de Steven W. Lingafelter (Systematic Entomolgy Laboratory, Plant Sciences Institute, ARS, USDA) quien mediante el análisis de fotografías de los especímenes corroboró la especie (Sánchez-Martínez et al., 2010).

\section{RESULTADOS Y DISCUSIÓN}

\section{Distribución y abundancia de especies forestales}

En los 60 sitios analizados se identificaron las siguientes especies de encinos: Quercus potosina, Q. eduardii, Q. side- roxyla, Q. rugosa, Q. resinosa, Q. chihuahuensis, Q. coccolobifolia, Q. laeta, Q. grisea y $Q$. microphylla, dentro de las 17 reportadas previamente para Aguascalientes (De la Cerda, 1989). Estas especies esporádicamente se encuentran en comunidades puras y por lo general se encuentran asociadas con cedros o táscates (Juniperus deppeana), madroños (Arbutus xalapensis y A. glandulosa), manzanita (Arctostaphylos pungens) y pinos (Pinus spp.). De las 10 especies identificadas, $Q$. potosina posee la distribución más amplia, habiéndose encontrado en $62 \%$ de los sitios muestreados. En el polígono analizado, los encinos rojos $Q$. sideroxyla y $Q$. eduardii poseen el segundo y tercer lugar, respectivamente, en su representación en el paisaje. Las especies con menor distribución son $Q$. coccolobifolia y $Q$. resinosa (Tabla 1).

De las especies de encinos identificadas en el polígono de estudio, tres son las que presentan una abundancia importante con una amplia representación en el paisaje forestal de la Sierra Fría. Asumiendo que los sitios muestreados son representativos del paisaje de la Sierra Fría, la abundancia máxima que una especie podría tener es 60 , eso significa que esa misma especie contribuye en un $100 \%$ a la diversidad de un sitio ecológico y eso se manifiesta en los 60 sitios. De esta manera, los encinos más abundantes en el paisaje de la Sierra Fría corresponden a $Q$. potosina, seguido en orden de importancia por los encinos rojos $Q$. eduardii y $Q$. sideroxyla. Los encinos blancos, $Q$. rugosa y $Q$. chihuahuensis, están bien representados, aunque con una distribución restringida a las barrancas y sitios húmedos. Las especies que presentan una población reducida son $Q$. coccolobifolia y $Q$. resinosa (Fig. 1). 
Tabla 1. Especies de encinos y su frecuencia en los muestreos realizados en la Sierra Fría, Aguascalientes. También se muestran las especies asociadas a cada especie de encino.

\begin{tabular}{|c|c|c|c|}
\hline Especie & Núm. de sitios & Frecuencia* $^{*}$ & Especies asociadas \\
\hline Quercus potosina Trel. & 37 & 61,6 & $\begin{array}{l}\text { J. deppeana, Arctostaphylos pungens, } \\
\text { Q. chihuahuensis, Pinus teocote }\end{array}$ \\
\hline Q. eduardii Trel. & 20 & 33,3 & $\begin{array}{c}\text { Q. sideroxyla, Q. rugosa, P. leiophyla, } \\
\text { Q. chihuahuensis }\end{array}$ \\
\hline Q. sideroxyla Humb. et Bonpl. & 14 & 23,3 & $\begin{array}{c}\text { Q. rugosa, P. leiophyla, } \\
\text { Q. chihuahuana, P. teocote }\end{array}$ \\
\hline Q. grisea Liemb. & 11 & 18,3 & $\begin{array}{c}\text { J. deppeana, Dodonaea viscosa, } \\
\text { A. pungens }\end{array}$ \\
\hline Q. rugosa Née & 7 & 11,6 & $\begin{array}{l}\text { P. leiophyla, Q. chihuahuensis, } \\
\text { Q. sideroxyla, J. deppeana }\end{array}$ \\
\hline Q. chihuahuensis Trel. & 7 & 11,6 & $\begin{array}{l}\text { Q. rugosa, Q. sideroxyla, P. teocote, } \\
\text { P. leiophyla, Q. eduardi, J. deppeana }\end{array}$ \\
\hline Q. laeta Liemb. & 5 & 8,3 & $\begin{array}{c}\text { J. depppeana, P. teocote, A, pungens, } \\
\text { P. leyophyla }\end{array}$ \\
\hline Q. microphyla Née & 3 & 5,0 & $\begin{array}{c}\text { Q. potosina, Q. sideroxyla, Q. rugosa, } \\
\text { Q. chihuahuensis }\end{array}$ \\
\hline Q. coccolobifolia Trel. & 2 & 3,3 & $\begin{array}{c}\text { P. lumholtzii, J. Deppeana, J. durangensis, } \\
\text { P. leiophyla, P. durangensis }\end{array}$ \\
\hline Q. resinosa Liemb & 1 & 1,0 & $\begin{array}{c}\text { Dodonaea viscosa, A. pungens, } \\
\text { Q. grisea }\end{array}$ \\
\hline
\end{tabular}

${ }^{*}$ Frecuencia $=($ No de sitios $)(100) /$ total de muestreos.

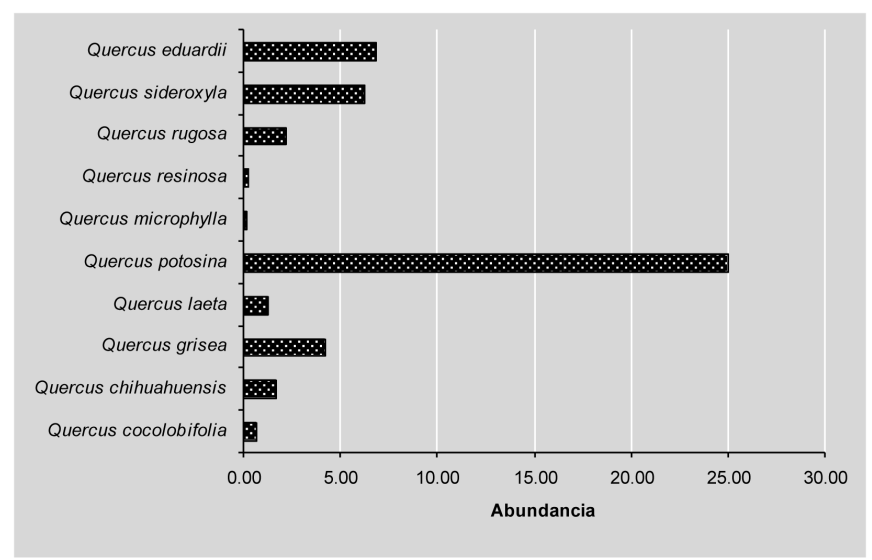

Figura 1. Abundancia de las especies de encinos identificadas en un polígono de 25 $\mathrm{km}^{2}$ en el ANP-Sierra Fría, Aguascalientes. El eje de las $X$ constituye el índice de abundancia, que oscila entre 0,1872 (Q. microphylla) y 25 (Q. potosina). El valor máximo del índice de abundancia podría ser 60 . 
Relación de las variables ambientales con la distribución y abundancia de especies forestales. Altitud y posición topográfica

El análisis de la distribución de los encinares en diferentes gradientes altitudinales sugiere que solamente una especie, Q. grisea, aparece en el primer estrato, aunque de manera aislada. Esta misma especie es abundante en el segundo estrato. Quercus potosina es la especie que más estratos altitudinales ocupa, pues se encuentra desde los 2200 hasta los $2800 \mathrm{msnm}$. Los estratos de $2400-2600$ y $2600-2800$, son donde mayor cantidad de especies se encuentran, probablemente debido a que en esta zona existe una mayor precipitación o a que las actividades de manejo (principalmente pastoreo) son distintas a las zonas planas y de altitudes menores. Especies como $Q$. resinosa, $Q$. coccolobifolia y $Q$. laeta, restringen su distribución a un solo estrato altitudinal (Tabla 2).

\section{Factores implicados en la distribución de especies forestales}

De acuerdo con el Análisis de Correspondencias Canónicas, existió una relación significativa entre las variables ambientales consideradas como responsables de la distribución de la vegetación y las especies de encinos detectadas (prueba

Tabla 2. Distribución altitudinal de las especies de encinos y sus principales especies asociadas en el área estudiada, dentro de la Sierra Fría, Aguascalientes.

\begin{tabular}{|c|c|c|c|c|c|c|c|c|c|c|c|c|c|c|c|c|}
\hline \multirow{3}{*}{ ESPECIES } & \multicolumn{16}{|c|}{ ALTITUD $(\mathrm{m} \times 1000)$} \\
\hline & \multicolumn{4}{|l|}{ A1 } & \multicolumn{3}{|l|}{ A2 } & \multicolumn{3}{|l|}{ A3 } & \multicolumn{3}{|l|}{ A4 } & \multicolumn{3}{|l|}{ A5 } \\
\hline & 1.9 & 2 & 2.1 & 2.19 & 2.2 & 2.3 & 2.39 & 2.4 & 2.5 & 2.59 & 2.6 & 2.7 & 2.79 & 2.8 & 2.9 & 3 \\
\hline \multicolumn{17}{|l|}{ Quercus potosina } \\
\hline \multicolumn{17}{|l|}{ Quercus resinosa } \\
\hline \multicolumn{17}{|l|}{ Quercus eduardii } \\
\hline \multicolumn{17}{|l|}{ Quercus rugosa } \\
\hline \multicolumn{17}{|l|}{ chihuahuensis } \\
\hline \multicolumn{17}{|l|}{ Quercus sideroxyla } \\
\hline \multicolumn{17}{|l|}{$\begin{array}{l}\text { Quercus } \\
\text { cocolobifolia }\end{array}$} \\
\hline \multicolumn{17}{|l|}{ Quercus grisea } \\
\hline Quercus laeta & & & & & & & & & & & & & & & & \\
\hline
\end{tabular}

NотA: las barras con franjas en gris indican que esa especie es abundante en los niveles altitudinales donde se le encontró. Por el contrario, las franjas en negro, indican que si bien, esa especie no es abundante, si se encontró en las altitudes marcadas, pero en bajas densidades. 
de Montecarlo $F=3,58 ; P=0,040)$. En el eje 1 las variables involucradas fueron la posición cóncava, la exposición norte, la fisiografía en bajo de ladera y la explotación dedicada principalmente a la cacería cinegética (explotación 7), variables relacionadas principalmente a sitios húmedos y barrancas. En el eje 2, las variables con mayor peso fueron las pendientes ligeras $(1 \%-5 \%)$ y explotaciones moderadas. El eje 3 mostró el menor peso solamente con una relación muy ligera entre la intensidad de manejo tipo 4 y las especies forestales incluidas en este eje. El eje 4 incorporó principalmente a variables relacionadas con el uso del terreno y la intensidad con que estas se realizan (Tabla 3).

Las especies de encinos identificadas se agruparon de acuerdo con las variables ambientales consideradas como responsables de su distribución y abundancia. De esta manera, Q. coccolobifolia es una especie que se distribuye entre los 2500 y los 2700 msnm, es raro encontrar esta especie en terrenos planos y con pendientes ligeras. Por otra parte, los encinos blancos ( $Q$. chihuahuensis y Q. rugosa), se distribuyen entre los 2400 y los 2600 msnm, en lugares con pendientes medias y ligeras, en exposiciones orientadas hacia el norte y con fisiografías en bajo de ladera. Los encinos rojos (Q. sideroxyla y $Q$. eduardii) se localizan en sitios con exposiciones norte, en laderas medias y bajas, en altitudes entre 2500 y $2700 \mathrm{msnm}$ y en sitios húmedos y con coberturas de dosel superiores a $50 \%$ (Tabla 4 ).

\section{Distribución de las enfermedades, identificación e incidencia de los fitopatógenos}

Phellinus robustus (Karst.) Bourd. et Galz., Phellinus gilvus (Schw.) Pat. y Phellinus everhartii (Ell. \& Gall.) A. Ames. Se identificaron tres especies de hongos basidiomicetos del género

Tabla 3. Coeficientes de correlación obtenidos entre los ejes canónicos y las variables ambientales, obtenidos mediante ACC en el paisaje de la Sierra Fría, en

Aguascalientes, Méx.

\begin{tabular}{lcccc}
\hline Variables ambientales & \multicolumn{4}{c}{ Correlaciones de las variables ambientales en los ejes canónicos } \\
\hline & Eje 1 & Eje 2 & Eje 3 & Eje 4 \\
\hline Cóncava & 0,8551 & & \\
Pendiente 5 & 0,655 & & \\
Exposición Norte & $-0,3099$ & & \\
Terreno plano & 0,6032 & & 0,5448 \\
Bajo de ladera & 0,3487 & & \\
Utilización 1 & & & \\
Intensidad 1 & & & \\
Intensidad 4 & 0,5405 & \\
Explotación1 & & & \\
Explotación 2 & 0,5314 & & \\
Explotación 7 & $-0,3629$ & & \\
Explotación Múltiple & & & \\
\hline
\end{tabular}




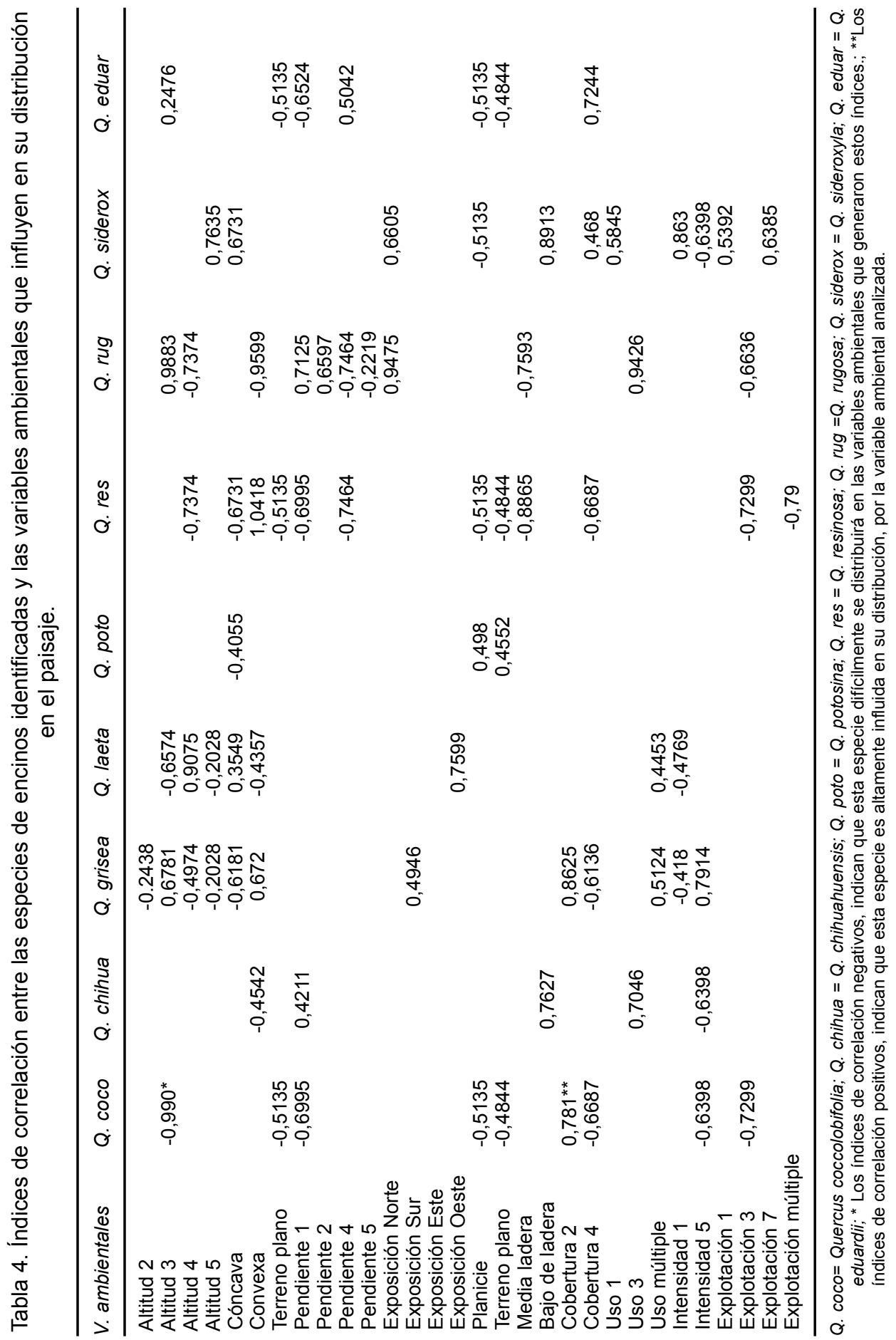


Phellinus: $P$. gilvus, $P$. robustus y $P$. everhartii, mismas que causaron pudrición blanca del duramen de los encinos y formación de cancros, de diversas longitudes y profundidades, al afectar la albura. Los basidiomas de $P$. robustus fueron localizados en dos encinos de $Q$. potosina, en la Barranca de Juan Francisco, del municipio de San José de Gracia, Aguascalientes. También fueron encontrados en dos árboles de $Q$. eduardii y en uno de $Q$. sideroxyla en Mesa del Águila del municipio de San José de Gracia, en un árbol de Q. Potosina, agonizando y caído, en la localidad Ilamada Huarache, municipio de Calvillo, Aguascalientes, y en un árbol de Q. potosina en Mesa de los Sapos del municipio de Rincón de Romos, Aguascalientes (Fig. 2a). Todos los árboles de encino fueron maduros $y$ estaban vivos. Los basidiomas de $P$. gilvus se encontraron sobre un ejemplar de $Q$. eduardii maduro y vivo en la comunidad El Ocote del municipio de Aguascalientes. Phellinus everhartii fue localizado en Mesa del Águila, San José de Gracia, en tres árboles maduros y vivos de $Q$. potosina y uno de Q. laeta (Fig. 2a). La incidencia de estas enfermedades varió de $0 \%$ a $15 \%$ (Fig. 2A). Las tres especies de Phellinus encontradas en este estudio fueron localizadas en árboles maduros. Al respecto, Swiecki y Bernhardt (1990) explican que el declinamiento de árboles maduros usualmente se asocia con la infección de diferentes hongos que pudren la madera, en el tronco, ramas principales $y / o$ en el sistema radicular, mismos que entran a través de heridas. Si el árbol es resistente y capaz de aislar la infección causada por el hongo, el patógeno puede ser detenido y la pudrición limitada. Si ocurre lo contrario, el hongo continúa la pudrición durante muchos años. Finalmente, desprovisto de su estructura y tejidos conductores de agua, el encino puede colapsar por su propio peso o deshidratarse en un periodo de alta demanda de agua y morir.

Ganoderma lucidum (W. Curt. ex Fr.) Karst. Este hongo basidiomiceto patógeno fue encontrado en árboles, jóvenes y maduros, vivos y muertos, de $Q$. potosina en La Angostura y Sierra Brava del municipio de Jesús María y en Los Alamitos y Mesa del Águila del municipio de San José de Gracia, causando pudrición blanca en la base del tallo y raíces de los encinos, así como la formación de cancros (Fig. 2b). La incidencia de la enfermedad causada por este patógeno fue de $0 \%$ a $10 \%$ (Fig. 2b). Cibrián et al. (2007), mencionan que este hongo afecta a coníferas y árboles latifoliados en los bosques naturales, pero que su importancia es reducida. A pesar de lo antes señalado, Swiecki y Bernhardt (1990) mencionan que $G$. lucidum no solo es un patógeno importante sino letal en encinos maduros, tanto en áreas silvestres como en zonas urbanas de California, EU. En California, Hickman y Perry (2003) señalan que este patógeno puede afectar a especies de los géneros Acacia, Pinus, Populus, Fraxinus y Salix, entre otros, y a los siguientes frutales: manzano, cerezo, cítricos, olivo, durazno, entre otros, a los cuales inclusive puede matar en un periodo de 3 a 5 años.

Hypoxylon thouarsianum (Lèveillè) Lloyd. Este hongo ascomiceto fue localizado sobre árboles jóvenes, maduros, vivos y muertos de $Q$. potosina, $Q$. rugosa, $Q$. sideroxyla y $Q$. eduardii, en Los Alamitos, La Congoja, Barranca de Juan Francisco, Mesa del Águila y Barranca Piletas del municipio de San José de Gracia; también en Mesa Tendida, Monte Grande, del municipio de Rincón de Romos; y en La Angostura, Barranca Verde, La Ciénega y El Huarache del municipio de Calvillo, Aguascalientes. La incidencia de este 


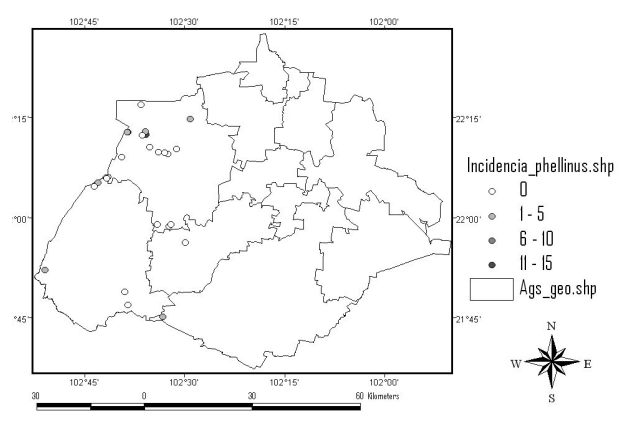

a)

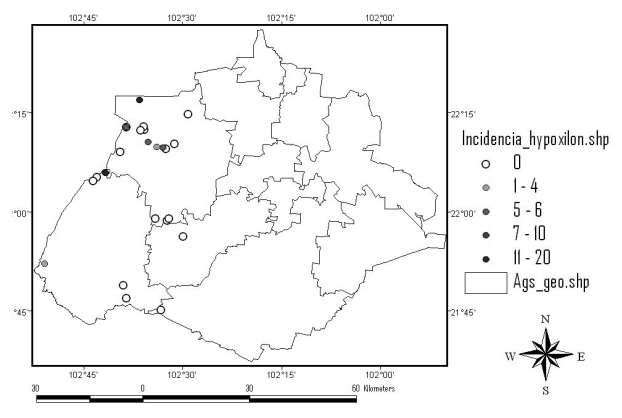

c)

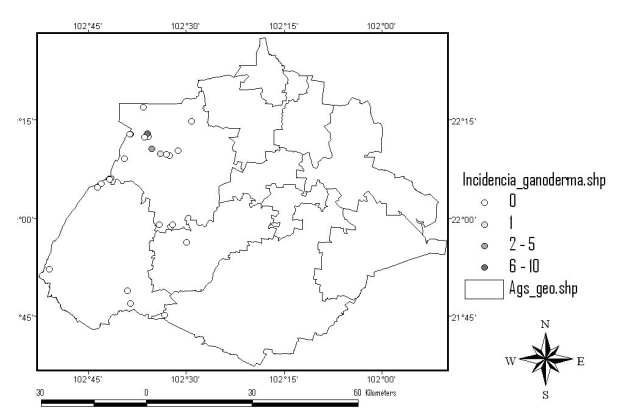

b)

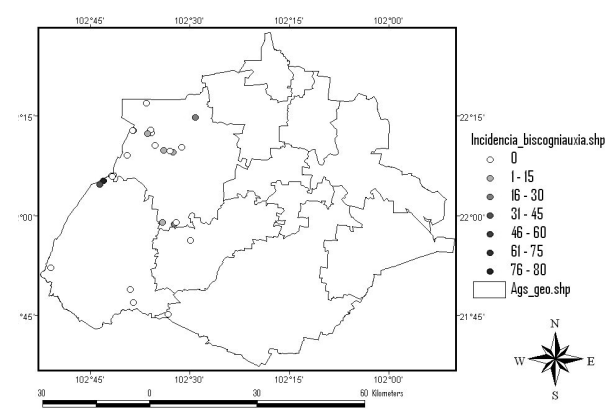

d)

Figura 2. Distribución e incidencia (\%) de a) Phellinus spp., b) G. Iucidum, c) $H$. thouarsianum y d) $B$. atropunctata en los encinares del estado de Aguascalientes.

Los archivos vectoriales fueron proporcionados por el Instituto Nacional de Investigaciones Forestales Agrícolas y Pecuarias.

hongo varió de $0 \%$ a $20 \%$ (Fig. 2c). Este hongo ascomiceto también causa pudrición blanca y cancros en los diferentes órganos de los árboles afectados. En un estudio realizado en la Sierra de Lobos, Guanajuato, Vázquez et al. (2004), encontraron que los principales agentes biológicos responsables de la declinación de los bosques de encino son el efecto combinado de Nectria galligena y $H$. thouarsianum, quienes actúan en sinergia causando una fuerte micosis con un comportamiento agresivo.
Biscogniauxia atropunctata (Schwein.: Fr.) Pouzar. Este hongo ascomiceto se encontró en árboles jóvenes, maduros, vivos y muertos, principalmente de $Q$. potosina en La Sauda del municipio de Jesús María, en Barranca Piletas y La Congoja del municipio de San José de Gracia, en El Tejamanil y El Pilar del municipio de Calvillo y Mesa de los Sapos del municipio de Rincón de Romos (Fig. 2d). Además, en menor grado, en árboles adultos de $Q$. eduardii. La incidencia de este hongo fue de $0 \%$ a $80 \%$. Este fitopa- 
tógeno causó pudrición blanca en ramas y tallos de los encinos. También forma cancros, al inicio pequeños, cubiertos por un polvo café que corresponde a las conidias de la fase asexual de Periconiella atropunctatum Sacc., posteriormente el hongo forma un estroma gris o plateado sobre los tejidos dañados que tienen una apariencia fibrosa, donde se forman los peritecios de la fase sexual (B. atropunctata). Cibrián et al. (2007) señalan que este hongo se ha encontrado asociado con la muerte de encinos, atribuida en algunos casos a Phytophthora cinnamomi, en Aguascalientes, Colima, Guerrero, Nayarit y Puebla.

\section{Agente causal de barrenación en encinos}

Durante el estudio se capturaron 40 ejemplares de insectos adultos, la mayoría de ellos obtenidos de las trampas instaladas en los fustes de los encinos, los cuales fueron identificados como Crioprosopus magnificus LeConte (Coleoptera: Cerambycidae) (Sánchez-Martínez et al., 2010) (figuras 3 y 4). Con excepción de un ejemplar que emergió de $Q$. eduardii, todos los especímenes emergieron de $Q$. potosina, por lo que se considera que esta especie es el principal huésped del insecto en el área de estudio. Detalles sobre la biología, incidencia y distribución geográfica de $C$. magnificus fueron publicados por Sánchez-Martínez et al. (2010).

\section{CONCLUSIONES}

Se identificaron 10 especies forestales arbóreas y arbustivas correspondientes al género Quercus. Quercus potosina y Juniperus deppeana son las más ampliamente distribuidas, ambas especies, en orden invertido, son las más abundantes. La altitud, los relieves (geoformas), la exposición solar y la fisiografía son las variables

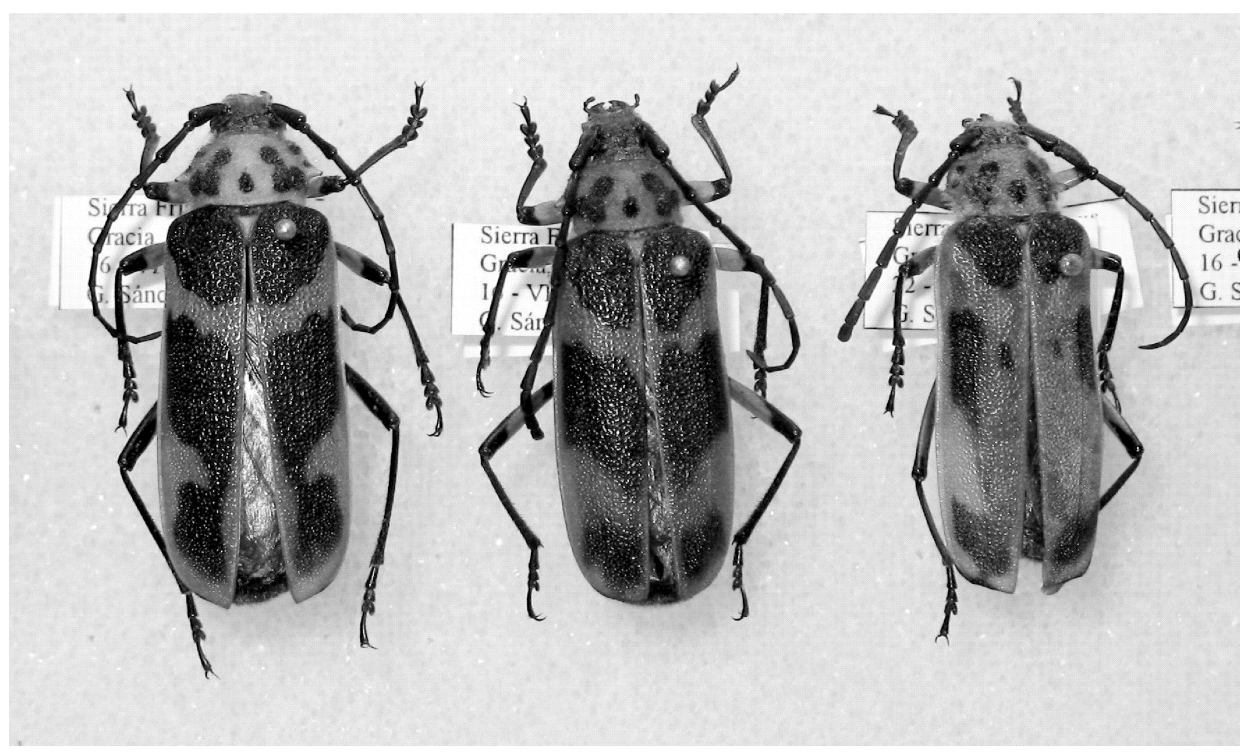

Figura 3. Ejemplares hembra de Crioprosopus magnifucus, emergidas de Quercus potosina en Sierra Fría, Aguascalientes. 


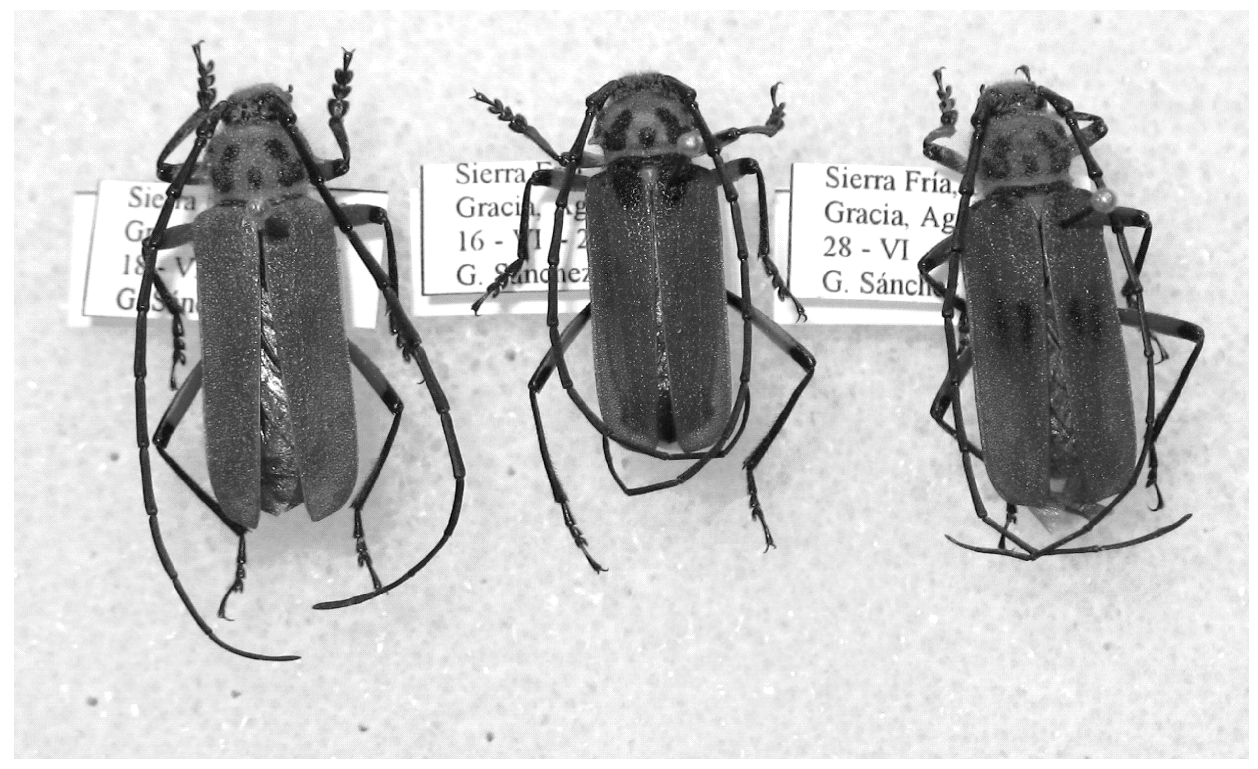

Figura 4. Ejemplares macho de Crioprosopus magnificus, emergidos de Quercus potosina en Sierra Fría, Aguascalientes.

que más influyen sobre la distribución y abundancia de la vegetación. Se sugiere profundizar la investigación a nivel regional, en especies cuya distribución es restringida a sitios muy específicos, tales como Q. coccolobifolia y Pinus lumholtzii. Se identificó a Phellinus robustus, $P$. gilvus y $P$. everhartii afectando encinos vivos y maduros. En encinos vivos, muertos, jóvenes o maduros se identificó a Ganoderma lucidum. Se identificaron también a los hongos oportunistas Hypoxylon thouarsianum y Biscogniauxia atropunctata en árboles estresados por factores abióticos o bióticos. Hypoxylon thouarsianum fue el hongo con mayor distribución en el paisaje. Quercus potosina es la especie frecuentemente más dañada. Se reportó la presencia del barrenador Crioprosopus magnificus en la Sierra Fría con una afectación que varía desde $0 \%$ hasta $75 \%$ de los encinos vivos. El periodo de emergencia de este insecto es en el mes de junio, a principios de la temporada de lluvia, y hay por lo menos dos generaciones traslapadas. El principal hospedero de C. magnificus es $Q$. potosina, Se recomienda abundar estudios sobre su biología, ecología, contribución en el declinamiento de los encinos y manejo bajo el contexto propio del ecosistema en que se encuentra.

\section{RECONOCIMIENTOS}

Este estudio fue apoyado financieramente por el Consejo de Ciencia y Tecnología del Estado de Aguascalientes (Fondos mixtos AGS-2005-C01-15087). Agradecemos al Instituto del Medio Ambiente del estado de Aguascalientes por el apoyo en transporte y personal de campo y a Sierra Fría, Aguascalientes A.C., por el apoyo logístico y las facilidades de trabajo en campo. Los autores 
reconocemos también el apoyo recibido por Francisco Juárez Reyes en el trabajo de campo para la sección de fitosanidad. Nahum Hernández, Alejandro Torres, Sergio Esparza, Luis Alberto Hernández, Diego Pérez Salicrup y Jorge Morfín, quienes asistieron en el levantamiento de información sobre ecología y distribución de los encinos. Se agradece a Clemente Villalobos por sus facilidades de hospedaje en Rancho Piletas.

\section{REFERENCIAS}

Alvarado R., D., L. de L. Saavedra R., A. Almaraz S., B. Tlapal B., O. Trejo R., J.M. Davidson, J.T. Kliejunas, S. Oak, J.G. O'Brien, F. Orozco T. y D. Quiroz R. 2007. Agentes asociados y su papel en la declinación y muerte de encinos (Quercus, Fagaceae) en el centrooeste de México. Polibotánica 23:1-21.

Alvarado-Rosales, D., L.L. Saavedra-Romero y A. Almaraz-Sánchez. 2008. Primer Reporte de Phytophthora cinnamomi Rands., asociado al encino (Quercus spp.) en Tecoanapa, Guerrero, México. Agrociencia 42(5):565-572.

Challenger, A. 1998. Utilización y conservación de los ecosistemas terrestres de México. Pasado presente y futuro. Comisión Nacional para el Conocimiento y Uso de la Biodiversidad, Instituto de Biología, UNAM, Agrupación Sierra Madre. México.

Cibrián T., D., J.T. Méndez-Montiel, R. Campos-Bolaños, H.O. Yates III y J.E. Flores-Lara. 1995. Insectos Forestales de México/Forest Insects of Mexico. Universidad Autónoma Chapingo. México, $391 \mathrm{p}$.

Cibrián T., D., R.D. Alvarado y D.S.E. García. 2007. Enfermedades Forestales en
México/Forest Diseases in Mexico. $\mathrm{UACH}$; Conafor-Semarnat, México; Forest Service USDA, EUA; NRCAN Forest Service, Canadá y Comisión Forestal de América del Norte, COFAN, FAO. Chapingo, México, 587 p.

De la Cerda L., M. 1989. Encinos de Aguascalientes. Universidad Autónoma de Aguascalientes. México, 84 p.

Espejel-Rodríguez, M.M.A., N. Santa CruzGarcía y M. Sánchez-Flores. 1999. El uso de los encinos en la región de La Malinche, estado de Tlaxcala, México. Bol. Soc. Bot. México 64:35-39.

Fierke, M.K., D.L. Kinney, V.B. Salisbury, D.J. Crook y F.M. Stephen. 2005. Development and comparison of intensive and extensive sampling methods and preliminary within-tree estimates of red oak borer (Coleoptera: Cerambycidae) in the Ozark Mountains of Arkansas. Environmental Entomology 34:184-192.

Gilbertson, R.L. y L. Ryvarden. 1986. North American Polypores. Vol. 1 Abortiporus-Lidtneria, Fungoflora Oslo. Noruega, 433 p.

Gilbertson, R.L. y L. Ryvarden. 1987. North American Polypores. Vol. 2 Abortiporus-Lidtneria, Fungoflora Oslo. Noruega, 452 p.

Govaerts, R. y D.G. Frodin. 1998. World checklist and bibliography of Fagales (Betulaceae, Corylaceae, Fagaceae and Tycodendraceae). Royal Botanical Gardens, Kew. Inglaterra, 407 p.

Heitzman, E., A. Grell, M. Spetich y D. Starkey. 2007. Changes in forest structure associated with oak decline in severely impacted areas of northern 
Arkansas. South. J. Appl. For. 31:1722.

Hickman, W.G. y E.J. Perry. 2003. Wood decay fungi in landscape trees. Pest Notes. University of California, UCIPM. USA. Publicación 74109, 4 p.

Hovore, F.T. 1983. Taxonomic and biological observations on southwestern Cerambycidae (Coleoptera). The Coleopterists Bulletin 37:379-38.

Ju-Ming J. y D.J. Rogers. 1996. A revision of the genus Hypoxylon. The Mycological Society of America. Memoir No. 20. APS Press. St. Paul, Minnesota, EUA, 382 p.

Kliejunas, J. 2005. Oak decline investigations in Mexico. A cooperative effort. USDA Forest Service, Forest Health Protection. Pacific Southwest Region, Vallejo. ht t p://www.google.com.mx/ search?hl=es\&site=Search\&q=Oak+de cline+investigations+in+Mexico. $+A+c o 0$ perative+effort.+USDA+Forest+Service $\% 2 \mathrm{C}+\&$ btnG=Buscar\&meta=

Krebs, C.K. 1993. Factors that limit distributions: Dispersal. In: C.K. Krebs (ed.), Ecology. John Whiley and Sons, pp. 41-56.

Lawrence, R., B. Moltzan y K. Moser. 2002. Oak decline and the future of Missouri's forests. Missouri Conservationist. July issue, pp. 1-8.

Nixon, K.C. 1993. The genus Quercus in Mexico. In: T.P. Ramammoorthy, R. Bye, A. Lot y J. Fa (eds.). Biological diversity of Mexico: origins and distribution. Oxford, University Press. Nueva York, pp. 447-458.

Nixon, K.C. 2003. Fagaceae. In: N. Smith, S.A. Mori, A. Henderson, D.W.
Stevenson y S. Heald (eds.). Families of Neotropical Flowering Plants. Princeton University Press. Princeton, N.J., pp. 156-158.

Ryvarden, L. 1991. Genera of Polypores. Nomenclature and taxonomy. Sinopsis Fungorum 5. Fungoflora, Oslo. Noruega, 349 p.

Ryvarden, L. 2000. Studies in Neotropical polypores 2: a preliminary key to Neotropical species of Ganoderma with a laccate pileus. Mycologia 92:180-191.

Sánchez-Martínez, G., O. Moreno-Rico y M.E. Siqueiros-Delgado. 2010. Crioprosopus magnificus LeConte (Coleoptera: Cerambycidae) in Aguascalientes, Mexico: biological observations and geographical distribution. Coleopterists Bulletin 64:319-328.

Solomon, J.D. 1995. Guide to insect borers in North American broadleaf trees and shrubs. USDA. Forest Service. Agriculture Handbook AH-706, pp. 294-476.

Swiecki, T.J. y E.A. Bernhardt. 1990. A Delicate balance: Impacts of diseases and insects on the health of California oaks. Fremontia 18:58-63.

Swiecki, T.J. y E.A. Bernhardt. 2006. A field guide to insects and diseases of California oaks. Gen. Tech Rep. PSWGTR-197. Albany, CA: Pacific Southwest Research Station, Forest Service, U.S. Department of Agriculture, 151 p.

Tainter, F.H., J.G. O’Brien, A. Hernández, F. Orozco y O. Rebolledo. 2000. Phytophthora cinnamomi as a cause of oak mortality in the state of Colima, Mexico. Plant Dis. 84(4):394-398. 